\title{
A MMSE Filter for Range Sidelobe Reduction
}

\author{
Nathan Goodman and Jim Stiles \\ The University of Kansas, Radar Systems and Remote Sensing Laboratory \\ 2291 Irving Hill Road, Lawrence, KS 66045 \\ Phone: (785) 864-7739, Fax: (785) 864-7789, E-mail: ngoodman@ rsl.ukans.edu
}

\begin{abstract}
In many cases, one needs to estimate a target that is obscured by the sidelobes of a very large, adjacent scatterer. For example, the Earth's surface can ruin nearsurface rain-rate measurements obtained from an airborne platform, or it can prevent a ground-penetrating radar from detecting land mines located at shallow depth. In these cases, the range sidelobes of the ground are larger than the targets near the surface. The range sidelobes cannot be optimally controlled because target information is not used. However, in most situations the location and magnitude of such a large scatterer can be predicted. In this paper, a minimum meansquared error (MMSE) estimator is proposed to improve the range sidelobe problem. The MMSE estimator uses information about the scenario to find the optimum processing filter. Simulated results are provided to demonstrate the effectiveness of this process.
\end{abstract}

\section{INTRODUCTION}

It is often desired that a radar detect a target located in close proximity to a large, specular scatterer. For example, downward-looking radars, as in [1], may need to detect targets just above the Earth, or ground-penetrating radars may need to detect targets buried just beneath the air-soil interface. Typically, the approach taken to this problem is to use as much bandwidth as possible [2-4] and to apply a window function to the frequency spectrum of the measurements. More signal bandwidth increases the effective number of range bins between the target and specular while windowing sacrifices some of that bandwidth for reduced sidelobes.

Although increased bandwidth certainly increases the resolution between two targets, a window function is rarely the optimum weighting function of choice for a weakly scattering target located in the first few sidelobes of a large scatterer. If a priori knowledge of the statistical properties of both targets is available, this information can be used to calculate the optimum weighting function that should be applied to the measurement spectrum. This optimum weighting function is calculated using the linear minimum mean-squared error (MMSE) estimator. Furthermore, the weighting function can be applied as part of the processing filter, rather than as weights on the data. In this way, the uniquely optimum weights for each range cell can be calculated and applied independently.

In this paper, the advantage of the MMSE estimator is confirmed through calculation and presentation of ambiguity functions. For two targets at different ranges from specular, the MMSE filter is calculated and correlated with the received spectrum as a function of range. The resulting ambiguity functions demonstrate the advantage of using MMSE estimation.

\section{BACKGROUND}

Strong scatterers can obscure nearby targets by their high sidelobes. For example, the first sidelobe of a uniform spectrum is the well-known $13 \mathrm{~dB}$ down from the mainlobe. Therefore, if the scattering from an adjacent range bin is more than $13 \mathrm{~dB}$ weaker than the target next to it, the weaker target is completely obscured by the sidelobe of the stronger. In order to alleviate this problem, windows are often applied to the data as a weighting function to reduce sidelobes at the cost of degraded resolution. Fig. 1 demonstrates a scattering profile where the range sidelobes of a large specular target dominate adjacent scatterers. In Fig. 2, a window function is applied, degrading resolution but reducing sidelobes below the level of some scatterers.

The measured data in the frequency domain are given by $r(f)$, and the expected return from the $\mathrm{i}^{\text {th }}$ range bin is given by $\rho_{i}(f)$. For the matched filter, the expected return is correlated with the measured data to get the estimate of the scattering from the $i^{\text {th }}$ range bin,

$$
\hat{\gamma}_{i}=\int r(f) \rho_{i}(f) d f .
$$

When a weighting function is applied, the estimate becomes

$$
\hat{\gamma}_{i}=\int w(f) r(f) \rho_{i}(f) d f .
$$

The weighting function, $w(f)$, is typically a window function chosen for the desired sidelobe level. However, in some cases, a priori information about the scattering profile may be available. For example, in airborne rain-rate profiling, the altitude of the airborne platform is known. Therefore, the range of the specular return from the Earth is known, and the expected RCS of the specular return may be known as well. In this case, a simple window function will not be the optimum choice for a weighting function given the information available. 


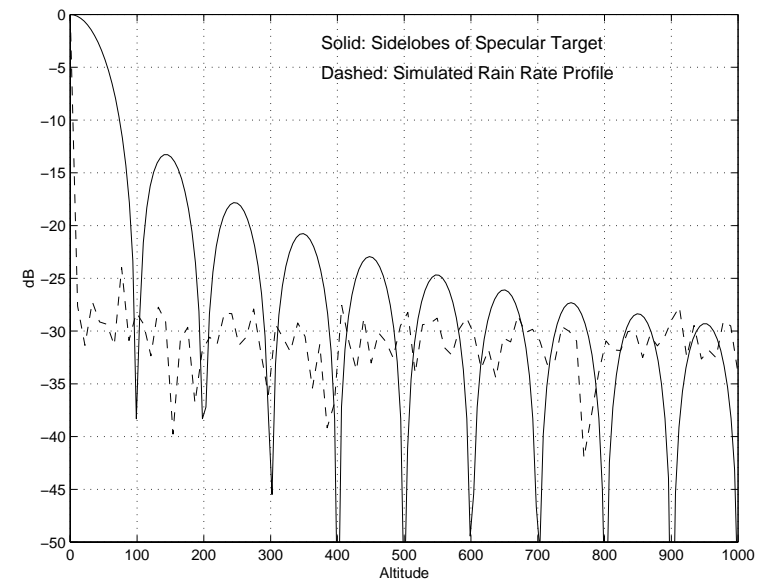

Fig. 1: High Range Sidelobes Obscure Weaker Scatterers

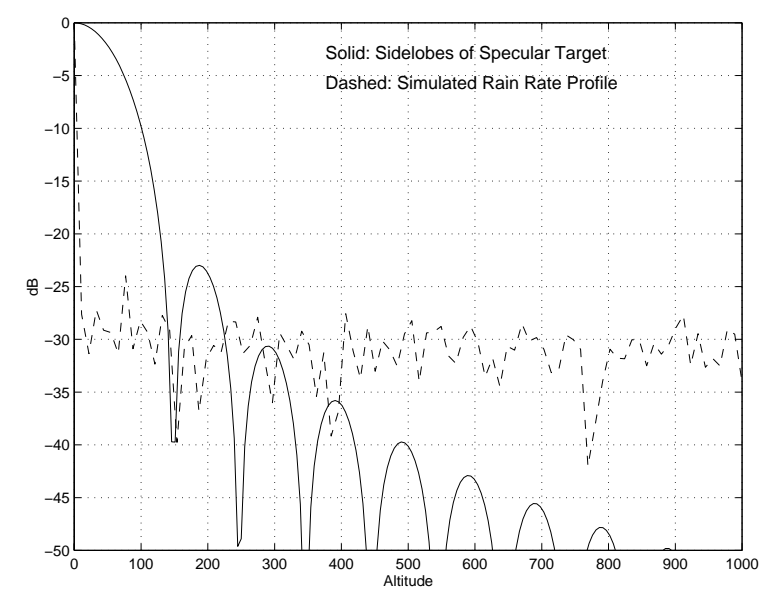

Fig. 2: Sidelobes Reduced with Weighting Function

The optimum, linear filter is the linear minimum meansquared error (MMSE) estimator. Taking the received data at discrete frequency points, a receive vector is formed given by,

$$
r(f)=\left[\begin{array}{llll}
r\left(f_{1}\right) & r\left(f_{2}\right) & \cdots & r\left(f_{M}\right)
\end{array}\right]^{\prime}
$$

The normalized, expected data from the $i^{\text {th }}$ range cell is similarly taken at these frequencies to get,

$$
\rho_{i}(f)=\left[\begin{array}{llll}
\rho_{i}\left(f_{1}\right) & \rho_{i}\left(f_{2}\right) & \cdots & \rho_{i}\left(f_{M}\right)
\end{array}\right]^{\prime} .
$$

If the complex RCS values of all range cells are given as,

$$
\boldsymbol{\gamma}=\left[\begin{array}{llll}
\gamma_{1} & \gamma_{2} & \cdots & \gamma_{N}
\end{array}\right]^{\prime}
$$

then the MMSE weighting function is given by,

$$
\mathbf{W}=\mathrm{E}\left[\boldsymbol{\gamma} \boldsymbol{\gamma}^{\prime}\right] \mathbf{P}^{\prime}\left(\mathrm{E}\left[\gamma \gamma^{\prime}\right] \mathbf{P} \mathbf{P}^{\prime}+\mathbf{K}_{n}\right)^{\sim 1},
$$

where $\mathbf{W}$ contains the weightings for each range cell, $\mathbf{P}$ contains the expected measurement vector for each range cell, $\mathbf{K}_{n}$ is the noise covariance matrix of the measurements, $\mathrm{E}[\cdot]$ denotes the expected value operation, and $(\square)^{\sim 1}$ denotes the pseudoinverse operation.

\section{SIMULATIONS}

The MMSE operator calculates the optimum linear weighting, and the improvement can be seen by studying the resulting ambiguity functions. Therefore, simulations have been performed to that end. The simulations presented here are for a downward, vertically looking radar with 10 range cells. The tenth range cell is centered at zero altitude. As a result, it contains the specular return from the Earth, and its scattering statistics are modified accordingly. A linear FM chirp was simulated in the frequency domain, and the elements of the matrix $\mathbf{P}$ were calculated by evaluating the response due to each frequency at multiple locations from the first to last range cells. The correlation between the MMSE weights and the measurements was then calculated and plotted. The correlation between $\cos ^{4}$ window weights and the measurements is also shown for comparison [5]. The magnitude of the signal spectrum was chosen to be uniform, and the bandwidth was such that a resolution of one range cell was obtained after weighting by the $\cos ^{4}$ window.

Fig. 3 shows the ambiguity function for a target in the third range cell. The scattering statistics in range cells one through nine are the same; therefore, the MMSE estimator uses that information to create approximately level sidelobes in this area. In the range cell with the specular target, however, a deep null is produced. While the $\cos ^{4}$ window has lower sidelobes in some areas, the MMSE weights optimally shape the ambiguity function according to target statistics. Therefore, not only is there a null on specular, but that null is optimally computed for the expected scattering statistics and noise estimates. Fig. 3 also shows three range cells beyond the air-ground interface. Because little scattering is expected from these range cells, the sidelobes are allowed to increase significantly.

Next, we show that the null on the specular target still exists when estimating the adjacent, or ninth, range cell. The window function, however, makes no distinction according to 




Fig. 3: Ambiguity Functions for Range Cell Far From Specular

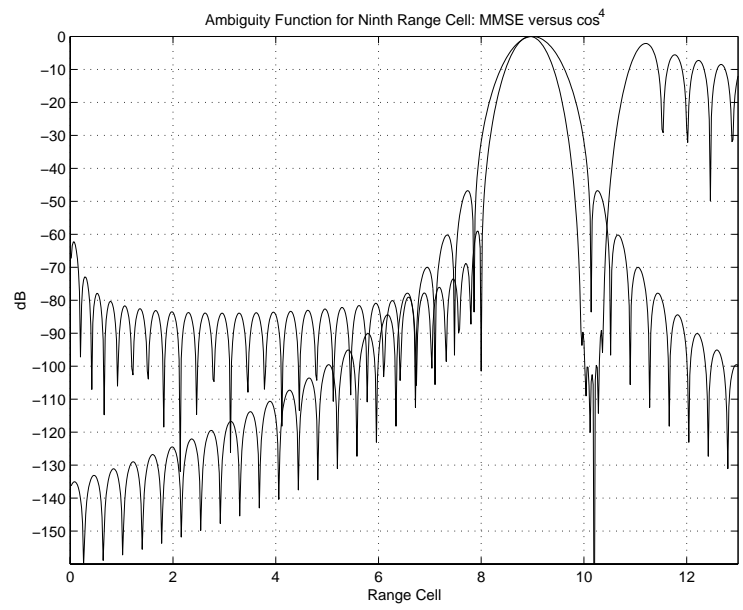

Fig. 4: Ambiguity Functions for Range Cell Adjacent to Specular

the proximity of large scatterers, so the high first and second sidelobes fall on the specular target. The comparison between MMSE and $\cos ^{4}$ for the ninth range cell, which is adjacent to the cell containing the ground return, is shown in Fig. 4.

Last, the MMSE weights as a function of SNR were investigated. From (6) is can be shown that the MMSE weights approach a scaled version of the matched filter as SNR decreases. Fig. 5 demonstrates this result. At the lowest SNR, the ambiguity function is seen to be the same form as what would be obtained from the matched filter response to a rectangular spectrum. Fig. 5 does not show, however, that the MMSE weights are smaller in magnitude than the matched filter by a constant value across the spectrum.
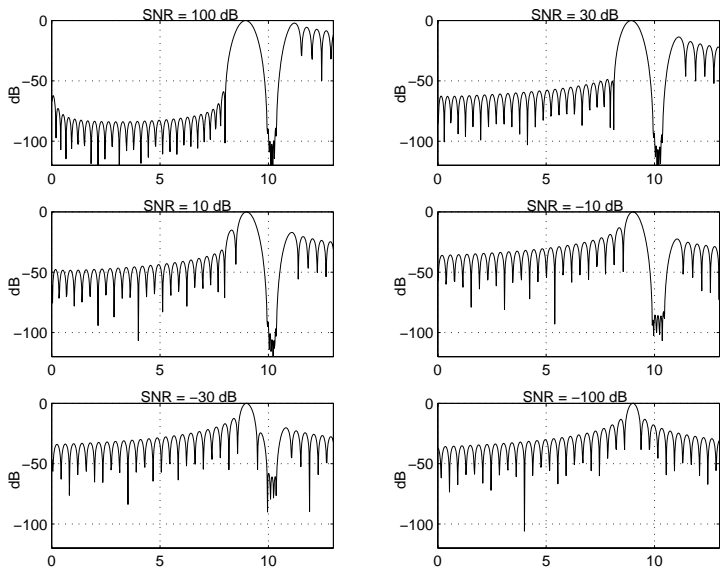

Fig. 5: Ambiguity Function for Various SNR Values

\section{CONCLUSIONS}

If statistical information is known a priori about a radar scene, that information can be used to compute a weighting function that is optimum for the scene. Rather than using a window function with a specific sidelobe level and sidelobe fall-off, the MMSE estimator forces sidelobes optimally tailored to the scene.

The advantage of this processing has been demonstrated through simulation. The simulations produced ambiguity functions that have been presented and show nulls where the specular return is located. Furthermore, the MMSE estimator takes the relative importance of that null versus SNR into account. This has been shown in another set of simulations where the MMSE estimator converged to a scaled version of the matched filter as SNR decreased.

\section{REFERENCES}

[1] A. R. Jameson, et al., "SIR-C/X-SAR Observations of Rain Storms," Remote Sensing of Environment, vol. 59, no. 2, pp. 267-279, Feb., 1997.

[2] J. Homer, H.T. Tang, I.D. Longstaff, "Radar imaging of shallow buried objects," Proc. 1999 IEEE International Geoscience and Remote Sensing Symposium, vol. 5, pp. 2477-2479, 1999.

[3] D. J. Daniels, "Ground Probing Radar Techniques for Mine Detection," Proc. $7^{\text {th }}$ International Conference on Ground-Penetrating Radar, vol. 1, pp. 313-317, May, 1998.

[4] B. Scheers and M. Piette, "Short-Pulse Response of Antipersonnel Landmines to UWB Signals," Proc. $7^{\text {th }}$ International Conference on Ground-Penetrating Radar, vol. 1, pp. 337-341, May, 1998.

[5] F. J. Harris, "On the Use of Windows for Harmonic Analysis with the Discrete Fourier Transform," Proc. of the IEEE, vol. 66, no. 1, Jan., 1978. 